Cochrane Database of Systematic Reviews

\title{
Magnetic seizure therapy for treatment-resistant depression
} (Protocol)

Jiang J, Li C, Chen Z, Cao X, Wang H, Li W, Wang J

Jiang J, Li C, Chen Z, Cao X, Wang H, Li W, Wang J.

Magnetic seizure therapy for treatment-resistant depression.

Cochrane Database of Systematic Reviews 2020, Issue 1. Art. No.: CD013528.

DOI: 10.1002/14651858.CD013528.

www.cochranelibrary.com 
TABLE OF CONTENTS

HEADER 1

ABSTRACT

BACKGROUND

OBJECTIVES

METHODS

ACKNOWLEDGEMENTS

REFERENCES

APPENDICES

CONTRIBUTIONS OF AUTHORS

DECLARATIONS OF INTEREST

SOURCES OF SUPPORT

\section{1}

2

3

3

7

8

10

11 
[Intervention Protocol]

\section{Magnetic seizure therapy for treatment-resistant depression}

Jiangling Jiang1, Chunbo Li ${ }^{1}$, Zhimin Chen ${ }^{1}$, Xinyi Cao ${ }^{1}$, Hongyan Wang ${ }^{1}$, Wei $\mathrm{Li}^{1}$, Jijun Wang ${ }^{2}$

1Shanghai Key Laboratory of Psychotic Disorders, Shanghai Mental Health Center, Shanghai Jiao Tong University School of Medicine, Shanghai, China. ${ }^{2}$ Department of EEG Source Imaging, Shanghai Mental Health Center, Shanghai Jiao Tong University School of Medicine, Shanghai, China

Contact address: Chunbo Li, Shanghai Key Laboratory of Psychotic Disorders, Shanghai Mental Health Center, Shanghai Jiao Tong University School of Medicine, 600 Wan Ping Nan Road, Shanghai, 200030, China. licb@smhc.org.cn, chunbo_li@163.com.

Editorial group: Cochrane Common Mental Disorders Group

Publication status and date: New, published in Issue 1, 2020.

Citation: Jiang J, Li C, Chen Z, Cao X, Wang H, Li W, Wang J. Magnetic seizure therapy for treatment-resistant depression. Cochrane Database of Systematic Reviews 2020, Issue 1. Art. No.: CD013528. DOI: 10.1002/14651858.CD013528.

Copyright @ 2020 The Cochrane Collaboration. Published by John Wiley \& Sons, Ltd.

\section{A B S T R A C T}

This is a protocol for a Cochrane Review (Intervention). The objectives are as follows:

To evaluate the effects of MST in comparison with sham-MST, antidepressant, or other non-pharmacological forms of treatments using electric or magnetic stimuli for adults with TRD. 


\section{B A C K G R O U N D}

\section{Description of the condition}

Major depressive disorder is characterised by a depressed mood and the loss of interest or pleasure, accompanied by a range of symptoms including weight loss or weight gain, insomnia or hypersomnia, psychomotor agitation or retardation, fatigue or loss of energy, feelings of worthlessness or inappropriate guilt, diminished ability to think or concentrate or decide, thoughts of death or suicidal ideation, or suicide attempts (APA 2013). Although antidepressants remain the first-line treatment for depressive disorders (NICE 2010), they only generate small- to medium-effect size when compared with placebo (Fournier 2010). Typically, we can recognise a situation where people with depressive disorders fail to adequately respond to multiple adequate antidepressant trials (in terms of dose, duration, and compliance) as treatment-resistant depression (TRD), but there is still no consensus on its definition (Trevino 2014).

Globally, depression is a common mental disorder, with an estimated 151 million people affected (Mathers 2008). Lifetime prevalence varies across nations, with an estimate of $14.6 \%$ in high-income countries and $11.1 \%$ in low-income countries (Bromet 2011). Rates of TRD vary from $30 \%$ to $60 \%$ depending on how the disorder is defined (Vieta 2011).

Noticeable personal, social, and economic morbidity as well as the loss of functioning and productivity often coexist with depression, and lead to substantial demands on service providers (NICE 2010). Depression is also associated with suicide (Kessler 2005), and increased rates of mortality (Cuijpers 2002). It is the second leading cause of disability globally, and it has contributed $8.1 \%$ of all-cause years lived with disability (Vos 2013). Depression contributes substantially to the burden of disease globally, and it ranks third worldwide, eighth in low-income countries, and first in middle-income and high-income countries (Mathers 2008). Compared with people with non-TRD depression, people with TRD experience much higher rates of long-term disability (Rizvi 2014), and economic burden (Mrazek 2014).

\section{Description of the intervention}

Magnetic seizure therapy (MST) is a potential alternative option to electroconvulsive therapy (ECT). For depression, ECT is effective, possibly even more so than medication therapy (UK ECT RG 2003). ECT is considered the last resort for people with depression with antidepressant intolerance, medication resistance, or other difficult-to-treat conditions, and can be a life-saving treatment in acute suicide-threatening and catatonic patients (Frederikse 2006). Nevertheless, ECT is often associated with cognitive adverse effects such as anterograde amnesia and postictal disorientation in the short-term, and retrograde amnesia in the long-term (Lisanby 2007). The rate of reported persistent memory loss varies between $29 \%$ and $55 \%$ (Rose 2003).

The hypothesis of using magnetic stimulation to induce therapeutic seizures arose in the mid-1990s (Sackeim 1994). The first successful and deliberate induction of seizures with magnetic pulses was conducted on two Macaca mulatta (rhesus macaque) in 2001 (Lisanby 2001a). Results from further animal experiments indicated that MST has a significantly lower impact on cognitive functions than electroconvulsive shock (ECS), the animal equivalent of ECT, and that no significant differences existed between MST and sham in most of the measures (McClintock 2013; Spellman 2008). There were no morphological changes or histological lesions found in postmortem animals that had received MST (Dwork 2009; Dwork 2014).

The first case report of MST on humans was published in 2001 , soon after the first MST report on animals (Lisanby 2001b). Since that time, several clinical trials have primarily investigated the feasibility, efficacy, and safety of MST. Lisanby and colleagues provided support for the feasibility of MST for depression and did not find evidence of serious adverse events (Lisanby 2003). Kayser and colleagues pointed out that MST has comparable antidepressant effects with ECT (Kayser 2011). Fitzgerald and colleagues claimed that MST has an antidepressant effect without apparent cognitive adverse effects (Fitzgerald 2013).

Usually, multiple sessions of MST are administered by trained psychiatrists twice or three times a week. The magnetic stimulation is delivered via a twin coil with its midline on the vertex or frontal cortex at $25 \mathrm{~Hz}$ to $100 \mathrm{~Hz}$. Given that the seizure threshold is likely to increase as treatment continues as ECT (Sackeim 1999), titration methods are employed to determine the dose of stimulation (100 pulses to 1000 pulses per session). In addition, MST is also administered under general anaesthesia.

\section{How the intervention might work}

The search for refining ECT techniques was impeded by a fundamental limitation, the electrical stimulus. The substantial impedance of the scalp and skull shuts most of the electrical stimulus away from the brain, resulting in the widespread stimulation of cortical and subcortical regions (Deng 2011; Rush 1968). In contrast, magnetic pulses, which can pass through the scalp and skull without resistance, are capable of focusing the stimulus on a specific area of the brain (Deng 2011). Additionally, magnetic stimulus can reach a depth of only a few centimetres, while electric currents are able to penetrate into deeper structures (Deng 2011). Theoretically, unlike ECT, MST can generate focus stimulation on superficial regions of the cortex, which may give MST the capability of producing comparable therapeutic benefits with the absence of apparent cognitive adverse effects. Indeed, some studies have provided evidence for this superiority of MST (Fitzgerald 2013; Polster 2015).

The effect of MST on brain glucose metabolism in depression has been investigated using positron emission tomography/ computer tomography (PET/CT) (Hoy 2013). Hoy 2013 found increased relative glucose metabolism in the basal ganglia, orbitofrontal cortex, medial frontal cortex, and dorsolateral prefrontal cortex. There was a trend difference of brain activation between responders and non-responders in the ventral anterior cingulate. Another study identifying MST's metabolic impact on receivers' brain via PET scans revealed an increase of glucose metabolism in the frontal cortex bilaterally and a decrease in the left striatum (Kayser 2015). These results indicate that the pattern of MST's antidepressant effects are consistent with the limbic-cortical dysregulation model of depression (Mayberg 2003).

\section{Why it is important to do this review}

Almost two decades after the first published reports of MST on people with depressive disorders, there is still insufficient 
information concerning its efficacy and safety. All published research is limited by the small number of participants and earlystage study design. Thus, high-quality evidence about the benefits and harms of MST are needed. As reflected by the trials registration in the International Clinical Trials Registry Platform (ICTRP) and ClinicalTrials.gov, many researchers and clinicians have recognised the potential of MST as an effective and less cognition-affected treatment for TRD. There will be a considerable number of highquality studies concerning the antidepressant effects of MST in the following decade. Meanwhile, it is important to reflect on what research has been currently performed in this area, what the limitations of these data are, and implications for future research. Therefore, synthesis of available evidence from up-todate, reliable, relevant, and critical trials is another best way to meet this demand. It may help clinicians and practitioners to make a decision about whether to offer people with difficult-totreat depression MST as an alternative treatment to ECT. However, there are currently no up-to-date systematic reviews specifically assessing the effectiveness of MST.

We will conduct the review according to this published protocol and report any deviations from it in the 'Differences between protocol and review' section of the systematic review.

\section{OB JECTIVES}

To evaluate the effects of MST in comparison with sham-MST, antidepressant, or other non-pharmacological forms of treatments using electric or magnetic stimuli for adults with TRD.

\section{METHODS}

\section{Criteria for considering studies for this review}

\section{Types of studies}

We will include randomised controlled trials (RCTs) including crossover RCTs and cluster RCTs. We will exclude quasi-RCTs.

\section{Types of participants}

\section{Participant characteristics}

We will include participants with TRD of both sexes, of any ethnicity, aged 18 years and older.

\section{Diagnosis}

The definition of TRD used in this review is a primary diagnosis of unipolar depression according to Diagnostic and Statistical Manual of Mental Disorders, 4th Edition (DSM-IV; APA 1994), Diagnostic and Statistical Manual of Mental Disorders, 4th Edition, Text Revision (DSM-IV-TR; APA 2000), Diagnostic and Statistical Manual of Mental Disorders, 5th Edition (DSM-5; APA 2013), International Classification of Diseases, 10th Revision (ICD-10; WHO 1992), and Chinese Classification of Mental Disorders (CCMD-3; CSP 2001), with no response or only partial response to at least four weeks of one or more antidepressants at their recommended doses. We will exclude participants who are resistant to only psychological treatments or other non-pharmacological treatments.

\section{Comorbidities}

We will include participants with comorbid non-psychotic mental health disorders and somatic illness as long as the comorbidity is not the focus of the study.

\section{Setting}

We will place no restrictions on the setting of studies.

\section{Types of interventions}

\section{Experimental intervention}

- MST (i.e. the magnetic induction of cerebral seizure activity after the intravenous induction of a brief general anaesthesia and the preadministration of a skeletal muscle relaxant drug). There will be no restrictions on the number or strength of doses.

\section{Comparator intervention}

- 'Sham-MST' or 'simulated-MST' (i.e. general anaesthesia without administering magnetic stimuli).

- ECT (i.e. the electric induction of cerebral seizure activity with or without brief general anaesthesia).

- Any type of antidepressant, regardless of its category, with or without antipsychotics.

- Other non-pharmacological forms of treatments using electric or magnetic stimuli, such as repetitive transcranial magnetic stimulation (rTMS) or transcranial direct current stimulation (tDCS).

\section{Types of outcome measures}

Studies that meet the above inclusion criteria will be included regardless of whether they report on the following outcomes.

\section{Primary outcomes}

- Symptom severity.

The primary outcome measure for assessing benefits will be symptom severity, determined from the following validated psychometric scales:

- continuous symptom scales, such as the Hamilton Rating Scale for Depression (HAMD) (Hamilton 1960), the Montgomery-Äsberg Depression Rating Scale (MADRS) (Montgomery 1979), the Clinical Global Inventory (CGI) (Guy 1976; Spearing 1997) and the Beck Depression Inventory (BDI) (Beck 1961), which will be analysed as continuous variables, or

- global state: clinically significant response in depressive symptoms (i.e. response, or non-response) as defined by the studies (short-term). For trials in which dichotomous outcome data are available, we will summarise these as the number of people who experienced those outcomes in each comparison group and the total number in each group, and will analyse them as dichotomous variables.

- Reduction in cognitive functions.

The primary outcome measure for assessing harms will be reduction in cognitive functions. These outcome measures of interest are changes in test scores and the rate of cognitive recovery by means of validated neuropsychological tests from baseline to the end of the course of MST. These will include (but will not be limited to): 
- Montreal Cognitive Assessment (MoCA);

- Repeatable Battery for Assessment of Neuropsychological Status (RBANS); and

- Cogstate computerised cognitive tests.

\section{Secondary outcomes}

- Suicides, suicide attempts, and self-harm.

- Suicides, measured as a dichotomous outcome (suicide versus no suicide).

- Suicide attempts, measured as a dichotomous outcome (suicide attempt versus no suicide attempt).

- Episodes of self-harm, measured as a dichotomous outcome (episodes of self-harm versus no episodes of self-harm).

- Quality of life.

Assessed using validated measures such as the Wisconsin Quality of Life Index (W-QLI; Becker 1993) and World Health Organization Quality of Life (WHOQOL; WHO 1998).

- Social functioning.

Measured using the Global Assessment of Functioning (GAF; APA 2000), or as defined by trialists (e.g. time to return to work or time to resumption of normal activities).

- Dropout for any reason.

Number of participants who dropped out during the trial as a proportion of the total number of randomised participants.

\section{- Serious adverse events.}

We will define serious adverse events as medical events that are life-threatening or that result in death, disability, or the significant loss of function, and that cause hospital admission or prolonged hospitalisation (e.g. cause-specific mortality, cerebral haemorrhage). These will be measured as a dichotomous outcome.

- Adverse events that lead to the discontinuation of treatment, measured as a dichotomous outcome.

\section{Timing of outcome assessment}

We will categorise outcomes as short term (up to six months from the beginning of treatment), medium term (six to 12 months) or long term (longer than 12 months). Short-term assessment will be our primary time frame. if a study reports more than one time point within one of the prespecified time frames, we will select the latest time point (e.g. measures at nine months rather than those at seven month will be included).

\section{Hierarchy of outcome measures}

If several continuous primary outcome measures are available, we will use results from the HAMD. If results from the HAMD are not available, we will use results from the BDI. However, if results from neither of the two are available, we will use results from the MADRS.

\section{Search methods for identification of studies}

In order to reduce publication and retrieval bias, we will not restrict our search by date, language, or publication status.

\section{Electronic searches}

A Cochrane Information Specialist will search the following biomedical databases and trial registers.

- Cochrane Common Mental Disorders Controlled Trials Register (CCMDCTR) (all available years).

- The Cochrane Central Register of Controlled Trials (CENTRAL) (the Cochrane Library, latest issue).

- Ovid MEDLINE (1946 to date of search).

- Ovid Embase (1974 to date of search).

- Ovid PsycINFO (all years to date of search).

- ISI Web of Science: Science Citation Index Expanded (SCIEXPANDED) (1970 to date of search).

- ISI Web of Science: Conference Proceedings Citation IndexScience (CPCl-S) (1990 to date of search).

- ClinicalTrials.gov (www.clinicaltrials.gov).

- World Health Organization International Clinical Trials Registry Platform (apps.who.int/trialsearch/).

The search strategies for the CCMDCTR, CENTRAL, and MEDLINE are listed in Appendix 1. The strategies will be adapted for the other databases as necessary. A detailed description of the Cochrane Common Mental Disorders Controlled Trials Register (CCMDCTR) can be found in Appendix 2.

\section{Chinese databases}

We will conduct complementary searches on the following Chinese biomedical databases, using the terms ("难治", "治疗抵抗", "顽 固性", "抑有症", "抑郁障碍", "情感障碍", "磁抽搐", "磁痉 挛").

- Chongqing VIP Database (VIP).

- Wanfang Database.

- China Hospital Knowledge Database (CHKD).

- Chinese Biology Medicine Database (SINOMED).

\section{Grey literature}

We will search the Networked Digital Library of Theses and Dissertations (NDLTD), Open Access Theses and Dissertations database (OATD), DART Europe e-theses Portal, Proquest's Dissertation and Thesis database (PQDT), OpenGrey, and Google Scholar for theses, dissertations, and other grey literature.

\section{Searching other resources}

We will check the reference lists of all included studies and relevant systematic reviews to identify additional studies missed from the original electronic searches (e.g. unpublished or in-press citations).

We will handsearch the Annual Meeting of American College of Neuropsychopharmacology (ACNP), Annual Scientific Convention and Meeting, and Annual Meeting of Europe College of Neuropsychopharmacology (ECNP) for conference proceedings.

We will contact trialists and subject experts for information on unpublished or ongoing studies or to request additional trial data. 


\section{Data collection and analysis}

\section{Selection of studies}

Two review authors (ZC, JJ) will independently perform the first assessment of titles and abstracts of all literature generated by electronic database searches for relevance. They will remove obviously irrelevant reports and will retrieve the full texts of the remaining literature. These two review authors will independently assess the full-text manuscripts against the inclusion criteria. As necessary, a third review author (WL) will act as an arbitrator to resolve disagreements that cannot be resolved through discussion by the two review authors. If usable data are included but not presented in the published manuscript of a study, we will contact the authors directly for further information. Review authors will not be blinded for articles' authorship, journals, and institutions. We will record reasons for exclusion in the 'Characteristics of excluded studies' table. At each time point, the numbers of studies selected will be detailed in a PRISMA flow diagram.

Where studies have multiple publications, we will collate the reports of the same study so that each study, rather than each report, is the unit of interest for the review, and give such studies a single identifier with multiple references.

\section{Data extraction and management}

Two review authors will independently extract data from studies using a data extraction form, which will be piloted on at least one trial included in the review. We will extract the following study characteristics:

- methods: study design, total duration of study, number of study centres and location, study setting, withdrawals, and date of study;

- participants: number, mean age, age range, gender, course of disease, severity of condition, diagnostic criteria, inclusion criteria, and exclusion criteria, co-morbid conditions;

- interventions: MST (coil placement, frequency, dose, number of sessions), ECT (electrode placement, pulse width, dose, number of sessions), concomitant medications, concomitant psychosocial interventions, and excluded medications;

- outcomes: primary and secondary outcomes specified and collected, time points reported, and whether the outcome data are reported in a usable way;

- notes: funding for trial, and notable conflicts of interest of trial authors.

As necessary, a third review author will act as an arbitrator to resolve disagreements that cannot be resolved through discussion by the two review authors. We will present these in the 'Characteristics of included studies' table.

\section{Main comparisons}

We will make the following main comparisons:

- MST versus sham-MST or simulated-MST;

- MST versus ECT;

- MST versus antidepressants;

- MST versus other non-pharmacological forms of treatments using electric or magnetic stimuli.

\section{Assessment of risk of bias in included studies}

Two review authors will independently assess the methodological bias of each trial according to the criteria in the Cochrane Handbook for Systematic Reviews of Interventions (Higgins 2011). The authors will not be blind to the authorship and source of the papers. Where inadequate details are provided, we will attempt to contact authors of the trial to obtain further information. We will settle any disagreements by consensus with the involvement of a third review author.

We will assess the risk of bias according to the following domains.

- Random sequence generation.

- Allocation concealment.

- Blinding of participants and personnel.

- Blinding of outcome assessment.

- Incomplete outcome data.

- Selective outcome reporting.

- Other bias.

We will judge each potential source of bias as high, low, or unclear and will provide a supporting quotation from the study report together with a justification for our judgement in the 'Risk of bias' table. We will summarise the risk of bias judgements across different studies for each of the domains listed. We will consider blinding separately for different key outcomes where necessary (e.g. for unblinded outcome assessment, the risk of bias for allcause mortality may be very different than for a participantreported pain scale). Where information on the risk of bias relates to unpublished data or correspondence with a trialist, we will note this in the 'Risk of bias' table.

When considering treatment effects, we will take into account the risk of bias for the studies that contribute to that outcome.

\section{Measures of treatment effect}

We will analyse continuous data using mean differences (MD) if studies use the same scales or using the standardised mean differences (SMD) if studies use different scales, with 95\% confidence intervals $(\mathrm{Cl})$. We will convert categorical data into dichotomous outcomes and calculate risk ratios (RR) and $95 \% \mathrm{Cl}$ for the individual studies. We will use Review Manager 5 for data analysis (Review Manager 2014).

\section{Unit of analysis issues}

\section{Cluster-randomised trials}

We will include cluster-randomised trials only if the following information is available.

- Number of clusters randomised to each intervention group or the mean number of each cluster (M).

- Outcome data ignoring the cluster design for the total number of participants (e.g. number or proportion of participants with events, or means and standard deviations (SD)).

- Intracluster (or intraclass) correlation coefficient (ICC) is provided or can be estimated.

An approximately correct analysis proceeds as suggested in the Cochrane Handbook for Systematic Reviews of Interventions (Section 16.3.4; Higgins 2011). The effective sample size of a single 
intervention group in a cluster-randomised trial is its original sample size divided by the 'design effect', which is $1+(M-1)$ ICC.

\section{Cross-over trials}

To avoid any carry-over effects, we will include only data from the first active treatment phase in the synthesis.

\section{Studies with multiple treatment groups}

In the case of trials with more than one treatment arm, we will include only relevant treatment arms and list other treatment arms in the 'Characteristics of included studies' table. If a study involves multiple relevant treatment arms (e.g. different magnetic field frequencies), we will try to combine them into a single group. We will summarise dichotomous outcomes data across groups and continuous outcomes into a single sample size, mean, and SD according to theCochrane Handbook for Systematic Reviews of Interventions (Section 7.7.3.8; Higgins 2011). If multiple relevant treatment arms cannot be combined (e.g. ECT and drugs as comparators), we will divide the sample size of the shared group so that the two arms can be treated as independent comparisons.

\section{Dealing with missing data}

We will contact investigators or study sponsors to obtain missing outcome data where possible. We will exclude a trial from the analysis if its outcome is missing for more than $40 \%$ of participants (Xia 2009). If SDs are unavailable from the trial authors, we will calculate missing SDs from reported standard errors, $\mathrm{P}$ values, or Cls using the methods described in the Cochrane Handbook for Systematic Reviews of Interventions (Sections 7.7.3.2 and 7.7.3.3; Higgins 2011) when possible.

We will contact study authors to obtain individual participant data where only a subset of participants (e.g. due to age or diagnosis) would be eligible. We employ the strategy which we use to deal with missing data, i.e., exclude a trial if less than $60 \%$ of participants are eligible.

\section{Assessment of heterogeneity}

We will assess heterogeneity of results across the included studies using the $1^{2}$ statistic (which provides an estimate of the percentage of inconsistency thought to be due to heterogeneity, with $30 \%$ to $60 \%$ representing moderate heterogeneity, $50 \%$ to $90 \%$ substantial heterogeneity, and $75 \%$ to $100 \%$ considerable heterogeneity (Higgins 2011)) and the $\mathrm{Chi}^{2}$ statistic. If the $\mathrm{I}^{2}$ statistic is greater than $50 \%$ or the $\mathrm{P}$ value of $\mathrm{Chi}^{2}$ is less than 0.10 , we will consider the results substantially heterogeneous (Higgins 2011).

We will visually inspect study characteristics and participant characteristics of all included studies, and data from individual studies to explore the possibility of heterogeneity.

\section{Assessment of reporting biases}

We will attempt to identify reporting bias by recording both trial outcomes planned in the protocol and outcomes actually reported. If there are discrepancies, we will try to obtain data on missing outcomes from authors of the study. We will perform funnel plots to assess reporting biases where 10 or more trials are included. Nevertheless, it must be noted that asymmetry in funnel plots can be caused by other methodological or sample size issues as well.

\section{Data synthesis}

We will use the random-effects model to calculate RR for dichotomous outcomes and MD or SMD for continuous outcomes for analyses after considering the potential heterogeneity in conducting trials and administering intervention. The randomeffects method incorporates the assumption that the different studies are estimating different intervention effects, and, therefore, is more conservative than the fixed-effect model. However, the random-effect model has the disadvantage of adding extra weight to small sample size trials, which often are the most biased ones (Higgins 2011). As a consequence, we will use the fixed-effect model in the sensitivity analysis to assess the robustness of findings. If there are differences between the two models, we will discuss what might be driving the difference (e.g. small-study effect). In addition, if data are considerably heterogeneous ( 12 of $75 \%$ or greater) (Higgins 2011), particularly if there is inconsistency in the direction of the effect and no clear reasons exist for heterogeneity, we will not undertake a meta-analysis. We will present the final data in descriptive form.

\section{Subgroup analysis and investigation of heterogeneity}

We will perform the following subgroup analyses for the primary outcomes (symptom severity and reduction in cognitive functions).

- High-dose MST (magnetic frequency $100 \mathrm{~Hz}$ or greater) versus low-dose MST (magnetic frequency less than $100 \mathrm{~Hz}$ ). Considerable efforts have been made for the development of MST with higher magnetic frequency (Hoy 2011). However, it is unclear whether it has superiority over low-dose MST.

- Long course (more than 12 sessions) versus short course (12 sessions or fewer). The number of treatments is expected to affect the outcomes, and there is no consensus on treatment schedules of MST, which leads to a broad range of numbers of treatments in trials (Hoy 2011).

- Differences in the definition of TRD (e.g. failure to respond to one, two, or three antidepressant agents). There is no consensus on the definition of TRD, which is expected to affect outcomes.

\section{Sensitivity analysis}

We will perform the following sensitivity analyses for the primary outcomes (symptom severity and reduction in cognitive functions) to examine the robustness of the effect size.

- Risk of bias: as risk of bias is a potential factor of influence on outcomes, we will use allocation concealment as a marker and exclude trials with a high and unclear risk of bias for allocation concealment.

- Attrition: we will exclude trials with a dropout rate of more than $20 \%$ to investigate the effects of the possible different outcomes of those participants who withdrew in each group.

- Missing data: we will exclude trials where the authors have imputed missing data to examine the potential differences between imputed values and actual values.

- Comorbidities: we will exclude trials where participants have comorbidities of other mental disorders to investigate the effects of comorbidities.

\section{'Summary of findings' table}

As recommended in the Cochrane Handbook for Systematic Reviews of Interventions (Section 11.5 and Section 12.2; Higgins 2011), we 
will employ GRADEprofiler (GRADEpro GDT 2015) to prepare the 'Summary of findings' table and the GRADE approach (Langendam 2013) to assess the quality of a body of evidence. We will justify all decisions to downgrade the quality of studies using footnotes and we will make comments to aid reader's understanding of the review where necessary.

We will include the following details in the 'Summary of findings' table.

- Participants or population: adults of either gender with a primary diagnosis of unipolar depression based on validated criteria and with no response or only partial response to at least four weeks of one or more antidepressants at their recommended doses.

- Settings: inpatient or outpatient clinical units/services.

- Intervention: MST, high dose or low dose, and long course or short course.

- Comparison: sham-MST or simulated-MST, ECT, antidepressants, and other non-pharmacological forms of treatments using electric or magnetic stimuli.
We will assess the following short-term outcomes for the 'quality' criteria.

- Continuous outcome measures for symptom severity.

- Categorical outcome measures for symptom severity.

- Reduction in cognitive functions.

- Quality of life.

- Social functioning.

- Dropout for any reason.

- Adverse events that lead to the discontinuation of treatment.

\section{ACKNOWLEDGEMENTS}

We thank the editorial team of the Cochrane Common Mental Disorders Group (CCMD) Group for providing guidance during protocol development.

CRG funding acknowledgement: the National Institute for Health Research (NIHR) is the largest single funder of the CCMD Group.

Disclaimer: the views and opinions expressed therein are those of the review authors and do not necessarily reflect those of the NIHR, the NHS or the Department of Health and Social Care. 


\section{RE F E R E N C E S}

\section{Additional references}

\section{APA 1994}

American Psychiatric Association. DSM-IV: Diagnostic and Statistical Manual of Mental Disorders. JAMA 1994;272(10):828-9.

\section{APA 2000}

American Psychiatric Association. Diagnostic and Statistical Manual of Mental Disorders, 4th Edition, Text Revision (DSM-IVTR). Washington (DC): American Psychiatric Association, 2000.

\section{APA 2013}

American Psychiatric Association. Diagnostic and Statistical Manual of Mental Disorders, 5th Edition (DSM-5). Washington (DC): American Psychiatric Association, 2013.

\section{Beck 1961}

Beck AT, Ward CH. An inventory for measuring depression. Archives of General Psychiatry 1961;4:561-71.

\section{Becker 1993}

Becker M, Diamond R, Sainfort F. A new patient focused index for measuring quality of life in persons with severe and persistent mental illness. Quality of Life Research 1993;2(4):239-51.

\section{Bromet 2011}

Bromet E, Andrade LH, Hwang I, Sampson NA, Alonso J, de Girolamo G, et al. Cross-national epidemiology of DSM-IV major depressive episode. BMC Medicine 2011;9(1):90.

\section{CSP 2001}

Chinese Society of Psychiatry. The Chinese Classification and Diagnostic Criteria of Mental Disorders Version 3 (CCMD-3). Jinan (China): Chinese Society of Psychiatry, 2001.

\section{Cuijpers 2002}

Cuijpers P, Smit F. Excess mortality in depression: a metaanalysis of community studies. Journal of Affective Disorders 2002; 72(3):227-36.

\section{Deng 2011}

Deng ZD, Lisanby SH, Peterchev AV. Electric field strength and focality in electroconvulsive therapy and magnetic seizure therapy: a finite element simulation study. Journal of Neural Engineering 2011;8(1):016007.

\section{Dwork 2009}

Dwork AJ, Christensen JR, Larsen KB, Scalia J, Underwood MD, Arango $\mathrm{V}$, et al. Unaltered neuronal and glial counts in animal models of magnetic seizure therapy and electroconvulsive therapy. Neuroscience 2009;164(4):1557-64.

\section{Dwork 2014}

Dwork AJ, Arango V, Underwood M, Ilievski B, Rosoklija G, Sackeim HA, et al. Absence of histological lesions in primate models of ECT and magnetic seizure therapy. American Journal of Psychiatry 2014;161(3):576-8.

\section{Fitzgerald 2013}

Fitzgerald PB, Hoy KE, Herring SE, Clinton AM, Downey G, Daskalakis ZJ. Pilot study of the clinical and cognitive effects of high-frequency magnetic seizure therapy in major depressive disorder. Depression and Anxiety 2013;30(2):129-36.

\section{Fournier 2010}

Fournier JC, De Rubeis RJ, Hollon SD, Dimidjian S, Amsterdam JD, Shelton RC, et al. Antidepressant drug effects and depression severity: a patient-level meta-analysis. JAMA 2010; Vol. 303, issue 1:47-53.

\section{Frederikse 2006}

Frederikse M, Petrides G, Kellner C. Continuation and maintenance electroconvulsive therapy for the treatment of depressive illness: a response to the National Institute for Clinical Excellence report. Journal of ECT 2006;22(1):13-7.

\section{GRADEpro GDT 2015 [Computer program]}

McMaster University (developed by Evidence Prime). GRADEpro GDT. Hamilton (ON): McMaster University (developed by Evidence Prime), 2015.

\section{Guy 1976}

Guy W. ECDEU Assessment Manual for Psychopharmacology. Rockville (MD): National Institute of Mental Health, 1976.

\section{Hamilton 1960}

Hamilton M. A rating scale for depression. Journal of Neurology, Neurosurgery, and Psychiatry 1960;23:56-62.

\section{Higgins 2011}

Higgins JP, Green S, editor(s). Cochrane Handbook for Systematic Reviews of Interventions Version 5.1.0 (updated March 2011). The Cochrane Collaboration, 2011. Available from handbook.cochrane.org. The Cochrane Collaboration.

\section{Hoy 2011}

Hoy KE, Fitzgerald PB. Magnetic seizure therapy for treatmentresistant depression. Expert Review of Medical Devices 2011;8(6):723-32.

\section{Hoy 2013}

Hoy KE, Thomson RH, Cherk M, Yap KS, Daskalakis ZJ, Fitzgerald PB. Effect of magnetic seizure therapy on regional brain glucose metabolism in major depression. Psychiatry Research 2013;211(2):169-75.

\section{Kayser 2011}

Kayser S, Bewernick BH, Grubert C, Hadrysiewicz BL, Axmacher N, Schlaepfer TE. Antidepressant effects, of magnetic seizure therapy and electroconvulsive therapy, in treatment-resistant depression. Journal of Psychiatric Research 2011;45(5):569-76.

\section{Kayser 2015}

Kayser S, Bewernick BH, Matusch A, Hurlemann R, Soehle M, Schlaepfer TE. Magnetic seizure therapy in treatment-resistant 
depression: clinical, neuropsychological and metabolic effects Psychological Medicine 2015;45(05):1073-92.

\section{Kessler 2005}

Kessler RC, Berglund P, Borges G, Nock M, Wang PS. Trends in suicide ideation, plans, gestures, and attempts in the United States, 1990-1992 to 2001-2003. JAMA 2005;293(20):2487-95.

\section{Langendam 2013}

Langendam MW, Akl EA, Dahm P, Glasziou P, Guyatt G, Schunemann HJ. Assessing and presenting summaries of evidence in Cochrane Reviews. Systematic Reviews 2013;2:81. [PUBMED: 24059250]

\section{Lisanby 2001a}

Lisanby SH, Luber B, Finck AD, Schroeder C, Sackeim HA. Deliberate seizure induction with repetitive transcranial magnetic stimulation in nonhuman primates. Archives of General Psychiatry 2001;58(2):199-200.

\section{Lisanby 2001b}

Lisanby SH, Schlaepfer TE, Fisch HU, Sackeim HA. Magnetic seizure therapy of major depression. Archives of General Psychiatry 2001;58(3):303-5.

\section{Lisanby 2003}

Lisanby SH, Luber B, Schlaepfer TE, Sackeim HA. Safety and feasibility of magnetic seizure therapy (MST) in major depression: randomized within-subject comparison with electroconvulsive therapy. Neuropsychopharmacology 2003;28(10):1852-65.

\section{Lisanby 2007}

Lisanby SH. Electroconvulsive therapy for depression. New England Journal of Medicine 2007;357(19):1939-45.

\section{Mathers 2008}

Mathers C, Fat DM, Boerma JT. The Global Burden of Disease: 2004 Update. Geneva: World Health Organization, 2008.

\section{Mayberg 2003}

Mayberg HS. Modulating dysfunctional limbic-cortical circuits in depression: towards development of brain-based algorithms for diagnosis and optimised treatment. British Medical Bulletin 2003;65(1):193-207.

\section{McClintock 2013}

McClintock SM, DeWind NK, Husain MM, Rowny SB, Spellman TJ, Terrace $\mathrm{H}$, et al. Disruption of component processes of spatial working memory by electroconvulsive shock but not magnetic seizure therapy. International Journal of Neuropsychopharmacology 2013;16(1):177-87.

\section{Montgomery 1979}

Montgomery SA, Asberg M. A new depression scale designed to be sensitive to change. British Journal of Psychiatry 1979;134(4):382-9.

\section{Mrazek 2014}

Mrazek DA, Hornberger JC, Altar CA, Degtiar I. A review of the clinical, economic, and societal burden of treatment-resistant depression: 1996-2013. Psychiatric Services (Washington, D.C.) 2014;65(8):977-87.

\section{NICE 2010}

National Collaborating Centre for Mental Health (UK). Depression: the Treatment and Management of Depression in Adults (Updated Edition). Vol. 90, Leicester (UK): British Psychological Society \& The Royal College of Psychiatrists, 2010.

\section{Polster 2015}

Polster JD, Kayser S, Bewernick BH, Hurlemann R, Schlaepfer TE. Effects of electroconvulsive therapy and magnetic seizure therapy on acute memory retrieval. Journal of ECT 2015;31(1):13-9.

\section{Review Manager 2014 [Computer program]}

Nordic Cochrane Centre, The Cochrane Collaboration. Review Manager 5 (RevMan 5). Version 5.3. Copenhagen: Nordic Cochrane Centre, The Cochrane Collaboration, 2014.

\section{Rizvi 2014}

Rizvi SJ, Grima E, Tan M, Rotzinger S, Lin P, McIntyre RS, et al. Treatment-resistant depression in primary care across Canada. Canadian Journal of Psychiatry 2014; Vol. 59, issue 7:349-57.

\section{Rose 2003}

Rose D, Fleischmann P, Wykes T, Leese M, Bindman J. Patients' perspectives on electroconvulsive therapy: systematic review. BMJ 2003;326(7403):1363.

\section{Rush 1968}

Rush S, Driscoll DA. Current distribution in the brain from surface electrodes. Anesthesia \& Analgesia 1968;47(6):717-23.

\section{Sackeim 1994}

Sackeim HA. Magnetic stimulation therapy and ECT. Convulsive Therapy 1994;10(4):255-8.

\section{Sackeim 1999}

Sackeim HA. The anticonvulsant hypothesis of the mechanisms of action of ECT: current status. Journal of ECT 1999;15(1):5-26.

\section{Spearing 1997}

Spearing MK, Post RM, Leverich GS, Brandt D, Nolen W. Modification of the Clinical Global Impressions (CGI) Scale for use in bipolar illness (BP): the CGI-BP. Psychiatry Research 1997;73(3):159-71.

\section{Spellman 2008}

Spellman T, McClintock SM, Terrace H, Luber B, Husain MM, Lisanby SH. Differential effects of high-dose magnetic seizure therapy and electroconvulsive shock on cognitive function. Biological Psychiatry 2008;63(12):1163-70.

\section{Trevino 2014}

Trevino K, McClintock SM, McDonald Fischer N, Vora A, Husain MM. Defining treatment-resistant depression: a comprehensive review of the literature. Annals of Clinical Psychiatry 2014;26(3):222-32. 


\section{UK ECT RG 2003}

UK ECT Review Group. Efficacy and safety of electroconvulsive therapy in depressive disorders: a systematic review and metaanalysis. Lancet 2003;361(9360):799-808.

\section{Vieta 2011}

Vieta E, Colom F. Therapeutic options in treatment-resistant depression. Annals of Medicine 2011;43(7):512-30.

\section{Vos 2013}

Vos T, Flaxman AD, Naghavi M, Lozano R, Michaud C, Ezzati M, et al. Years lived with disability (YLDs) for 1160 sequelae of 289 diseases and injuries 1990 - 2010: a systematic analysis for the Global Burden of Disease Study 2010. Lancet 2013;380(9859):2163-96.

\section{WHO 1992}

World Health Organization. The ICD-10 Classification of Mental and Behavioural Disorders: Clinical Descriptions and Diagnostic Guidelines. Geneva: World Health Organization, 1992.

\section{WHO 1998}

World Health Organization. The World Health Organization Quality of Life Assessment (WHOQOL): development and general psychometric properties. Social Science \& Medicine 1998;46(12):1569-85.

\section{Xia 2009}

Xia J, Adams C, Bhagat N, Bhagat V, Bhoopathi P, El-Sayeh H, et al. Losing participants before the trial ends erodes credibility of findings. Psychiatrist 2009;33(7):254-7.

\section{APPENDICES}

\section{Appendix 1. Search strategies}

1. Cochrane Specialised Register (CCMDCTR) cross-search (all available years):

("magnetic seizure therapy" or "magnetic therapy" or MST:ab)

2.CENTRAL (the Cochrane Library, current issue):

\#1 MST:ab

\#2 "magnetic seizure" or "seizure therapy" or "magnetic therapy"

\#3 "affective disorder" or depress* or TRD

\#4 (\#1 or \#2) and \#3

3. Ovid MEDLINE (1946 to date of search):

1 Depressive Disorder, Treatment-Resistant/

2 (depress $^{\star}$ adj3 (refractory ${ }^{\star}$ or resistan ${ }^{\star}$ or relaps ${ }^{\star}$ or recurr ${ }^{\star}$ or chronic ${ }^{\star}$ or persist ${ }^{\star}$ or sever or $\left.^{\star}\right)$.ti,ab,kf.

3 (depress $^{\star}$ and ((antidepress ${ }^{\star}$ or SSRI ${ }^{\star}$ or SNRI ${ }^{\star}$ or (serotonin adj3 (uptake or reuptake or re-uptake)) or medication* or psychotropic or treatment* or respon*) adj2 fail*)).ti,ab,kf.

4 (depress $^{\star}$ and ((antidepress* or SSRI ${ }^{\star}$ or SNRI ${ }^{\star}$ or (serotonin adj3 (uptake or reuptake or re-uptake)) or psychotropic medication* or treatment*) adj2 ("no respon*" or "not respon*" or nonrespon* or non-respon* or unrespon*))).ti,ab,kf.

5 (depress $^{\star}$ and (augment ${ }^{\star}$ or potentiat $\left.\left.{ }^{\star}\right)\right)$.mp.

6 TRD.ab.

7 (or/1-6)

8 (magnetic seizure or seizure therapy or magnetic therapy).ti,ab,kf.

9 MST.ab.

10 (8 or 9$)$

11 magnetic seizure therapy.ti.

12 ((7 and 10$)$ or 11$)$

13 randomized controlled trial.pt.

14 controlled clinical trial.pt.

15 random*.ab.

16 (placebo or sham or mock or fake or dummy).ti,ab,kf.

17 double-blind ${ }^{\star} . t i, a b, k f$.

18 (crossover ${ }^{\star}$ or cross over ${ }^{\star}$ ).ti,ab,kf.

19 group?.ab.

20 RCT.ab.

21 (trial or study).ti,ab,kf.

22 exp animals/ not humans.sh.

23 (or/13-21) not 22

2412 and 23

\section{Appendix 2. Cochrane Specialised Register (CCMDCTR)}

\section{Cochrane Common Mental Disorders Controlled Trials Register (CCMDCTR)}


The Cochrane Common Mental Disorders Group maintains an archived specialised register of randomised controlled trials (RCT), the CCMDCTR. This register contains over 40,000 reference records (reports of RCTs) for anxiety disorders, depression, bipolar disorder, eating disorders, self-harm, and other mental disorders within the scope of this Group. The CCMDCTR is a partially studies-based register with about $50 \%$ of reference records tagged to about 12,500 individually PICO-coded study records. Reports of trials for inclusion in the register are collated from (weekly) generic searches of MEDLINE (from 1950), Embase (from 1974), and PsycINFO (from 1967), quarterly searches of the Cochrane Central Register of Controlled Trials (CENTRAL), and review-specific searches of additional databases. Reports of trials are also sourced from international trial registries; drug companies; and handsearching of key journals, conference proceedings, and other (non-Cochrane) systematic reviews and meta-analyses. Details of CCMD's core search strategies (used to identify RCTs; $\mathrm{cmd}$.cochrane.org/ search-strategies-identification-studies) can be found on the Group's website with an example of the core Medline search displayed below.

A weekly search alert based on condition and RCT filter only

\section{1. [MeSH Headings]:}

eating disorders/ or anorexia nervosa/ or binge-eating disorder/ or bulimia nervosa/ or female athlete triad syndrome/ or pica/ or hyperphagia/ or bulimia/ or self-injurious behavior/ or self mutilation/ or suicide/ or suicidal ideation/ or suicide, attempted/ or mood disorders/ or affective disorders, psychotic/ or bipolar disorder/ or cyclothymic disorder/ or depressive disorder/ or depression, postpartum/ or depressive disorder, major/ or depressive disorder, treatment-resistant/ or dysthymic disorder/ or seasonal affective disorder/ or neurotic disorders/ or depression/ or adjustment disorders/ or exp antidepressive agents/ or anxiety disorders/ or agoraphobia/ or neurocirculatory asthenia/ or obsessive-compulsive disorder/ or obsessive hoarding/ or panic disorder/ or phobic disorders/ or stress disorders, traumatic/ or combat disorders/ or stress disorders, post-traumatic/ or stress disorders, traumatic, acute/ or anxiety/ or anxiety, castration/ or koro/ or anxiety, separation/ or panic/ or exp anti-anxiety agents/ or somatoform disorders/ or body dysmorphic disorders/ or conversion disorder/ or hypochondriasis/ or neurasthenia/ or hysteria/ or munchausen syndrome by proxy/ or munchausen syndrome/ or fatigue syndrome, chronic/ or obsessive behavior/ or compulsive behavior/ or behavior, addictive/ or impulse control disorders/ or firesetting behavior/ or gambling/ or trichotillomania/ or stress, psychological/ or burnout, professional/ or sexual dysfunctions, psychological/ or vaginismus/ or Anhedonia/ or Affective Symptoms/ or *Mental Disorders/

\section{2. [Title/Author Keywords]:}

(eating disorder ${ }^{\star}$ or anorexia nervosa or bulimi* or binge eat* or (self adj (injur ${ }^{\star}$ or mutilat ${ }^{\star}$ )) or suicide* or suicidal or parasuicid ${ }^{\star}$ or mood disorder ${ }^{\star}$ or affective disorder ${ }^{\star}$ or bipolar i or bipolar ii or (bipolar and (affective or disorder ${ }^{\star}$ )) or mania or manic or cyclothymic ${ }^{\star}$ or depression or depressive or dysthymi* or neurotic or neurosis or adjustment disorder* or antidepress* or anxiety disorder* or agoraphobia or obsess ${ }^{\star}$ or compulsi ${ }^{\star}$ or panic or phobi* or ptsd or posttrauma* or post trauma* or combat or somatoform or somati\#ation or medical ${ }^{\star}$ unexplained or body dysmorphi* or conversion disorder or hypochondria* or neurastheni* or hysteria or munchausen or chronic fatigue* or gambling or trichotillomania or vaginismus or anhedoni^ or affective symptoms or mental disorder ${ }^{\star}$ or mental health).ti,kf.

\section{3. [RCT filter]:}

(controlled clinical trial.pt. or randomized controlled trial.pt. or (randomi\#ed or randomi\#ation).ab,ti. or randomly.ab. or (random adj3

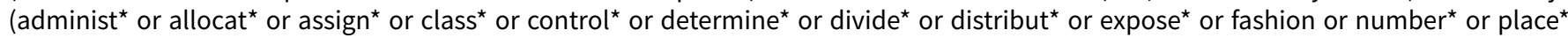

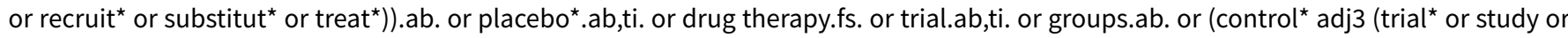
studies)).ab,ti. or ((singl* or doubl* or tripl* or trebl*) adj3 (blind ${ }^{\star}$ or mask ${ }^{\star}$ or dummy $\left.\left.{ }^{\star}\right)\right) . m p$. or clinical trial, phase ii/ or clinical trial, phase iii/ or clinical trial, phase iv/ or randomized controlled trial/ or pragmatic clinical trial/ or (quasi adj (experimental or random)).ti,ab. or ((waitlist ${ }^{\star}$ or wait* list $^{\star}$ or treatment as usual or TAU) adj3 (control or group)).ab.)

\section{4. (1 and 2 and 3$)$}

Records were screened for reports of RCTs within the scope of the Cochrane Common Mental Disorders Group. Secondary reports of RCTs were tagged to the appropriate study record. Similar weekly search alerts were also conducted on Ovid Embase and PsycINFO, using relevant subject headings (controlled vocabularies) and search syntax, appropriate to each resource. The Group's Specialised Register fell out of date with the Editorial Group's move from Bristol to York in the summer of 2016.

\section{CONTRIBUTIONS OF AUTHORS}

All review authors participated in the preparation of the protocol.

\section{DECLARATIONSOF INTEREST}

JJ: none.

CL: none.

XC: none.

HW: none. 
WL: none.

JW: none.

\section{SOURCES OF SUPPORT}

\section{Internal sources}

- Shanghai Mental Health Center, Shanghai Jiao Tong University School of Medicine, China.

\section{External sources}

- Research Grant from Shanghai Hospital Development Center (SHDC12014111); Science and Technology Commission of Shanghai Municipality (14411961400, 13dz2260500), Shanghai Health System Leadership in Health Research Program (XBR2011005), China. 